\title{
Filigrane
}

Écoutes psychanalytiques

\section{Présentation. Qu'est la psychanalyse devenue ? Partie 2}

\section{Sophie Gilbert}

Volume 23, numéro 2, automne 2014

Qu'est la psychanalyse devenue ? Partie 2

URI : https://id.erudit.org/iderudit/1028919ar

DOI : https://doi.org/10.7202/1028919ar

Aller au sommaire du numéro

Éditeur(s)

Revue Santé mentale au Québec

ISSN

1192-1412 (imprimé)

1911-4656 (numérique)

Découvrir la revue

Citer ce document

Gilbert, S. (2014). Présentation. Qu'est la psychanalyse devenue ? Partie 2.

Filigrane, 23(2), 7-11. https://doi.org/10.7202/1028919ar d'utilisation que vous pouvez consulter en ligne.

https://apropos.erudit.org/fr/usagers/politique-dutilisation/ 


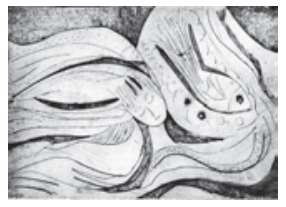

\section{Présentation. Qu'est la psychanalyse devenue? partie 2}

\section{Sophie Gilbert}

ors d'un colloque s'étant déroulé à Montréal en novembre 2013, nous - avons — rappelons-le — invité plusieurs cliniciens des milieux institutionnels à répondre à la question «qu'est la psychanalyse devenue?». Ce deuxième numéro accordé à ce thème nous donne l'occasion de publier la suite de ces conférences, devenues articles.

Large question, toujours d'actualité, le devenir de la psychanalyse nous interpelle non seulement à partir des milieux institutionnels - ce qui interroge la place de la psychanalyse en ces milieux aujourd'hui bombardés d'approches toujours plus "efficaces» — mais aussi, jusque dans nos bureaux privés. Que faire de la demande des consultants (et de celle des instances "politicoéconomiques» représentées par les cadres d'un système de santé et de services sociaux) mais aussi, comment la psychanalyse peut-elle prétendre répondre à des problématiques considérées nouvelles, ou du moins, qui sont davantage diagnostiquées dans notre contexte occidental?

Si nos auteurs ne répondent pas directement à ces questions, ils nous donnent toutefois des outils pour alimenter notre réflexion; que ce soit en se référant à ce qui constitue la base d'une approche psychanalytique, ou encore, en prônant et démontrant les vertus d'ouverture et d'inscription franche dans le contexte spatiotemporel de notre époque... je vous l'accorde: peut-être en rechignant un peu au départ car après tout, qui aime à ce point sortir de sa «zone de confort»? Mais rappelons ici combien l'époque du confort de la psychanalyse est révolue. Pour le pire? À cet égard, certes, plusieurs témoignages de cliniciens en milieux institutionnels se sont avérés saisissants, voire choquants, devant l'ampleur du dénigrement dont fait l'objet cette approche jugée dépassée (car bien sûr, pour certains, il n'existe que Freud... et l'Edipe: voilà en bref la psychanalyse que se targuent de connaître les psychiatres nouvelle génération!). En découlent des pressions importantes pour se conformer — voire se recycler — dans une nouvelle 
approche thérapeutique probante, ou encore, une mise à l'écart progressive, puisque les diagnostics (je répète: les diagnostics, car le patient, l'individu existe à peine dans notre réseau de services psys) dictent une approche thérapeutique particulière (ou un traitement, c'est selon!) de la maladie, dans une clinique surspécialisée en fonction de ce même diagnostic. Quelles sont les réactions que nous pouvons avoir à cette nouvelle réalité? Comment peut-on justifier la pertinence de la compréhension puis de l'approche psychanalytique sans se positionner en victimes, opprimés, mais tout de même en défendant ce qui nous apparaît comme les forces de cette approche, l'actualité aussi de celle-ci surtout lorsque l'on se réfère à tant d'auteurspsychanalystes-théoriciens contemporains qui ont révolutionné (ou même, en quelque sorte, qui ont «ouvert») notamment notre vision du cadre des interventions psychanalytiques? N’y a-t-il pas urgence de reconsidérer notre position de psys, dans un monde qui éclate de partout? Si la troisième guerre mondiale à laquelle nous assistons est celle du danger toujours voilé (que symbolisent si bien les drones), de l'inconnu voire du méconnu (comment comprendre les conflits liés aux peuples islamiques sans se référer à l'histoire), de l'extrémisme des agirs et de la pensée, de la violence en direct et sans filtre aucun révélée par les nouvelles technologies, du désespoir de générations entières de jeunes prêts à tout pour donner un sens à leur vie devant l'insensé d'un quotidien dont on oublie trop souvent (ou l'on cherche à oublier) l'inhumanité? Si les interventions d'urgence sur les lieux de séismes, de guerre ou d'actes terroristes demeurent la place incontestée des psys, comment utiliser dans la société le savoir sur l'humain, progressivement développé par la psychanalyse, la compréhension singulière et en profondeur du sujet - de sa violence, de ce qui échappe à sa conscience, de son manque fondamental, de sa dualité sous toutes ses formes (double message, double en soi et inquiétante étrangeté, double manifeste-latent, ambivalence, conflits, etc.) ? Ce sont là autant de pistes pour envisager, nous semble-t-il, le devenir de la psychanalyse. Ajoutons, sans ironie et avec toute notre sympathie: Je suis Elsa?

Trop peu abordée sous cet angle, peut-être, la question de l'avenir de la psychanalyse vient interroger les diverses modalités de sa transmission. Cette question apparaît bien sûr difficile à évoquer lorsque l'on considère l'ampleur des débats, des conflits et des scissions, ayant traversé les milieux 
psychanalytiques à ce propos. Cette question de fond, qui touche notamment les institutions et associations de psychanalystes, sous-tend celle de la légitimité du titre de psychanalyste (question à la mode, au Québec et ailleurs), mais aussi celles non moins polémiques de la place laissée à la jeunesse au sein de ces associations, des modalités et exigences de la formation, et de la place de la psychanalyse et de son enseignement en milieu universitaire. Nous avons l'occasion d'ailleurs dans ce numéro d'approfondir le thème de la transmission de la psychanalyse en milieu universitaire - ce qui est la raison d'être de notre rubrique «Psychanalyse à l'université».

Du reste, la mise en cause de l'ouverture des milieux psychanalytiques aux nouvelles générations, de même que des modalités de la transmission tant de la théorie que de la pratique psychanalytique par le biais des associations vient nous positionner autrement dans le présent débat. D’une posture de victime, elle nous pousse à nous poser en acteurs, par un questionnement qui ne peut avoir lieu qu'à l'interne et que l'on pourrait reformuler ainsi: «Qu'avons-nous fait de la psychanalyse?».

Notre expérience est que cette question trouve sa place, aujourd'hui, dans certains milieux; en résonance peut-être avec les écrits qui se multiplient à ce propos: quelle est la place des psychanalystes dans leur propre devenir? Le dernier en liste à notre connaissance, est le travail audacieux signé Sébastien Dupont $^{1}$; l'auteur n'y va pas de main morte, et s'il peut parfois sembler accusateur, au final, le lecteur demeure avec plusieurs voies (réalistes) pour l'avenir de la psychanalyse, à commencer par une ouverture à l'altérité. C'est d'ailleurs ce qui ressort du propos de nos auteurs: autres approches psychothérapeutiques, autres domaines de la connaissance, autres milieux d'intervention, autres dimensions - culturelle, artistique — du sujet, altérité en soi (qui fonde en quelque sorte la créativité), etc. Ne serait-ce pas là le plus fertile des «retours à Freud», à une humanité toute freudienne, à une inscription culturelle dans son époque et les milieux scientifique, culturel et humaniste qui y étaient en vogue. Un tel retour se veut toutefois non pas un enterrement, mais une inspiration à aller de l'avant!

En ouverture de notre dossier, Jean-Charles Crombez répond à notre question avec toute la créativité qu'on lui connaît. À partir d'une vignette, voire d'une anecdote issue de son travail (dans une perspective psychosomatique - où le corps et la psyché sont à la fois conviés dans l'intervention) en 
milieu hospitalier, il tente de dégager les ingrédients nécessaires pour qu'une intervention s'avère psychanalytique, notamment — mais pas seulement — dans les pratiques en milieu institutionnel.

Patricia Riaño aborde ensuite notre thème sous l'angle de son expérience personnelle de psychanalyste en institution. Elle nous rappelle que la soumission à un cadre institutionnel amène le psychanalyste à user de créativité, afin de respecter certaines conditions inhérentes à la pratique analytique, tout en évitant de priver - dans une logique puriste - la clientèle de ses services. Ainsi, elle situe son travail à court terme dans la perspective des rencontres préliminaires, avec les limites qui s'en suivent mais aussi, la richesse et l'ouverture qui peuvent en découler pour le sujet.

Pour clore notre série d'articles succédant au colloque de 2013, Carole Levert témoigne de sa façon toute singulière de côtoyer d'autres approches institutionnelles en santé mentale. Elle y relève ce qui met en cause la psychanalyse, puis questionne la place que la psychanalyse cherche à occuper dans l'institution, et plus généralement dans le social et auprès des autres professions. De son propos ressort la spécificité de l'écoute «orientée par la psychanalyse», de même qu'un rapport moins hiérarchique que de réciprocité à tisser avec d'autres professions ou sphères d'activité qui véhiculent des valeurs plus humaines qu'économiques.

Dans la rubrique «Heteros», François Duparc nous convie à une exploration des plus pertinentes du travail psychanalytique, sous l'angle particulier du concept central de la pensée. Le lecteur est amené à faire le lien avec une pratique clinique où la demande première porte sur des symptômes au premier plan, qui se situent justement hors pensée, dans l'impensable, voire l'informe... Un article aussi intéressant du point de vue clinique que de celui du développement psychique et des assises fantasmatiques de celui-ci.

Par la suite, Alexandre L'Archevêque et Élise Bourgeois-Guérin élaborent sur l'oralité inhérente à la psychose, en lien avec leur pratique spécifique de psychologues en milieu communautaire, dans une communauté thérapeutique montréalaise, la Chrysalide. Leur objectif consiste à pousser plus loin la théorie de même que les fondements psychanalytiques d'une pratique clinique en milieu de vie, ce qui s'intègre tout à fait dans l'esprit de ce numéro où sont interrogés notamment les lieux où la psychanalyse peut s'inscrire, en 2014, hors cadre traditionnel.

Il est intéressant de remarquer que nos articles de la rubrique «Psychanalyse à l'université» répondent eux aussi en quelque sorte à l'interrogation de notre thème. Pour survivre, la psychanalyse n'a-t-elle pas intérêt à 
se développer également dans ce lieu — universitaire - de transition, en parallèle voire en partenariat avec les associations de psychanalystes?

En ce sens, Pascal Roman et ses collaboratrices partagent avec notre lectorat une façon originale de transmettre - et pas seulement enseigner la psychanalyse à l'université, dans le cadre d'un cours d'introduction qui permet aux étudiants de faire l'expérience pratique non seulement des concepts-clés, mais également de tisser des liens entre clinique et processus de création. Dans un autre ordre d'idées, Irène Krymko-Bleton aborde la spécificité de l'analyse du discours dans le cadre de recherches psychanalytiques universitaires. En résulte une approche novatrice de la recherche qui se veut tributaire non seulement de la théorie psychanalytique, mais également de l'apport non négligeable des linguistes à l'élaboration du sens au travers d'un matériau de prédilection de la connaissance du sujet humain: la parole. Ces deux articles nous ramènent à une dimension essentielle, semble-t-il, du devenir de la psychanalyse: la poursuite de son ouverture (amorcée par Freud, notamment) aux autres disciplines, artistiques autant que linguistiques.

Nous terminons ce numéro avec une entrevue réalisée par Laurence Branchereau auprès de Roland Gori, lors de son dernier passage à Montréal. Il est intéressant de constater que son choix fut d'aborder l'avenir de la psychanalyse, et ce, sans lien direct avec notre argumentaire. De fait, ses derniers écrits abordent plus ou moins directement cette large question sous différents angles, dont celui de l'épistémologie - entre sciences dites exactes et sciences humaines. De plus, selon une perspective socioculturelle (et même politique), il interroge le statut de l'individu, du sujet humain, de la santé et du bien-être, en lien avec ce «reste» (cette place de l'inconnu, du non savoir), cette interstice dans lequel l'écoute psychanalytique est appelée à s'immiscer.

\section{Note}

1. S. Dupont, L'autodestruction du mouvement psychanalytique, Paris, Gallimard, 2014. 